\title{
Katzenallergene auch in der Antarktis
}

\begin{abstract}
Mit allergischen Symptomen bei gegen Hausstaubmilben und Katzen sensibilisierten Patienten muß offensichtlich auch an den entlegensten Orten der Welt gerechnet werden. Allergene dieser Tiere fand man nun sogar in katzen- und milbenfreien Orten wie der Antarktis - zumindest dort, wo sich auch Menschen aufhalten.
\end{abstract}

Daß vor allem Allergene von Hauskatzen bei uns weit verbreitet sind und in relevanten Konzentrationen auch in katzenfreien Wohnungen und öffentlichen Gebäuden nachzuweisen sind, ist bekannt. Eine Forschergruppe untersuchte nun die Behausungen des ständig bewohnten Basislagers Scott Base auf Ross Island an der Grenze zur Antarktis auf das Vorhandensein des Hauptallergens der Hausstaubmilbe, Der $\mathrm{p}$ 1, und der Hauskatze, Fel

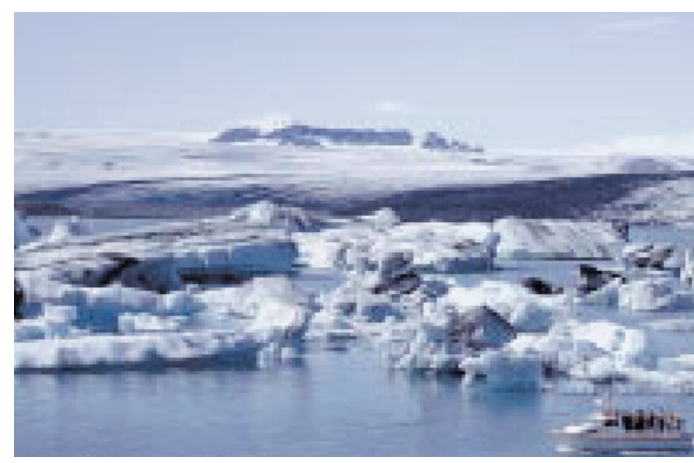

Unberührte Wildnis der Antarktis - doch wo der Mensch ist, sind auch Katzenallergene nicht weit. d 1. Das Besondere an der kleinen Studie: Für die Hausstaubmilben ist das Klima dort zu trocken und zu kalt, um sich gut vermehren zu können und die Antarktis gilt als katzenfrei.

Trotzdem wurden die Forscher bei ihrer Allergensuche fündig. Zwar hielten sich die Konzentrationen des Hausstaubmilbenallergens in geringen Grenzen, und ein positiver Nachweis gelang überwiegend in der Bekleidung von 11 gerade im Basislager eingetroffenen Menschen.

\section{Fel d 1-Konzentrationen bis zu $4 \mu \mathrm{g} / \mathrm{g}$ Staub}

Verbreiteter war das Katzenallergen Fel d 1 - immerhin 7 der 11 frisch eingetroffenen Personen gaben an, zu Hause eine Katze zu halten. Das Allergen wurde in einer Konzentration von $0,44 \mu \mathrm{g} / \mathrm{g}$ Staub in einem Aufenthaltsraum gefunden und in 7 von 12 Matratzen in einer Konzentration zwischen $0,43 \mu \mathrm{g} / \mathrm{g}$ und $1,49 \mu \mathrm{g}$. Noch höhere Werte fand man in der Bekleidung. 10 von 11 Pullovern wiesen Konzentrationen von $0,45 \mu \mathrm{g} / \mathrm{g}$ bis $\mathrm{zu} 4,18 \mu \mathrm{g} / \mathrm{g}$ Staub auf.

Die Untersuchung belegt noch einmal die weite passive Verbreitung von wichtigen Innenraumallergenen durch den Menschen. Dabei bleibt vor allem das Katzenallergen auch über weite Strecken in hoher Konzentration intakt erhalten und scheint keinem natürlichen Abbau unterworfen zu sein (siehe dazu auch das Editorial von G. Schultze-Werninghaus in Allergo Journal 1/99, S. 1).

Eine vollständige Allergenkarenz kann wohl an keinem Ort der Welt mehr erwartet werden - auch wenn sich die Autoren der Studie über die klinische Relevanz ihrer Befunde ausschweigen. Aktuelle Studien (Munir et al.; 1997) gehen aber von einem Sensibilisierungspotential durch Fel d 1 bei einer Allergenexposition von weniger als $0,5 \mu \mathrm{g} / \mathrm{g}$ Staub aus.

$b k$

Siebers R, Weinstein Ph, Fitzharris P, Crane J. House-dust mite and cat allergens in the Antarctic. Lancet 1999; 353: 1942.

\section{Klimatherapie: Therapeutische Langzeiteffekte}

Die Hochgebirgsklimatherapie (HGKT) gilt als bewährte Form der Ganzheitsmedizin z.B. bei Erkrankungen des atopischen Formenkreises mit Neurodermitis, Asthma bronchiale oder allergischer Rhinokonjunktivitis. Die Klimatherapie in einer weitgehend allergenarmen Umgebung beeinflußt den ganzen Menschen auf physischer wie psychischer Ebene. Neben einem Soforteffekt hat diese Therapieform auch einen meßbaren Langzeiterfolg.

Eine Fragebogenuntersuchung, bei der die Angaben von insgesamt 11451 Patienten ausgewertet wurden, belegt dies eindrucksvoll. Die Befragten litten überwiegend an verschiedenen Atopieformen und hatten einen vier- bis sechswöchigen Aufenthalt in der Alexanderhausklinik in Davos absolviert.

Über $90 \%$ der Patienten waren bei ihrer Entlassung bezüglich der Hauptdiagnose beschwerdefrei oder ihr Zustand wesentlich verbessert. Auch die Langzeiteffekte überzeugen: Bei allen Patienten nahm die Häufigkeit der Krankheitsschübe nach dem Aufenthalt deutlich ab, der Hautbefund stabilisierte sich nachhaltig. Die Zahl der Tage mit Arbeitsunfähigkeit reduzierte sich ebenso wie der Bedarf vor allem an externen Kortikoiden.

Die Ergebnisse belegen eine langanhaltende Steigerung der Lebensqualität von Allergikern durch die Klimatherapie. Auch die Kosten amortisieren sich, wenn man die Einsparung teurer Therapeutika wie auch den deutlichen Rückgang an Arbeitsunfähigkeitstagen berücksichtigt. $b k$

Drzimalla K, Wagner SA, Disch R. Langzeitergebnisse der Hochgebirgsklimatherapie in Davos. Allergologie 1999; 22/Supplement 1: S29-S35. 